Abstract 0011/\#287 Table 1 Clinicopathologic characteristics of organ-confined p53abn vulvar squamous cell carcinoma by margin status

\begin{tabular}{|c|c|c|c|c|c|c|c|c|c|}
\hline \multirow{3}{*}{ Group } & \multirow{2}{*}{\multicolumn{2}{|c|}{ Total }} & \multirow{2}{*}{\multicolumn{2}{|c|}{$\begin{array}{c}\text { (i) } \\
\text { dVIN } \\
\text { margin + }\end{array}$}} & \multirow{2}{*}{\multicolumn{2}{|c|}{$\begin{array}{c}\text { (ii) } \\
\text { p53abn } \\
\text { margint }\end{array}$}} & \multirow{2}{*}{\multicolumn{2}{|c|}{$\begin{array}{c}\text { (iii) } \\
\text { Margins clear }\end{array}$}} & \multirow{3}{*}{$p^{*}$} \\
\hline & & & & & & & & & \\
\hline & $n$ & (\%) & $n$ & (\%) & $\mathrm{n}$ & (\%) & $\mathrm{n}$ & $(\%)$ & \\
\hline Total & 57 & $100.0 \%$ & 16 & $28.1 \%$ & 11 & $19.3 \%$ & 30 & $52.6 \%$ & \\
\hline Follow-up (years, median) & 4.5 & & 4.1 & & 3.8 & & 5.3 & & $1^{*}$ \\
\hline Age [years, median, (SD)] & 78.8 & $(12.5)$ & 75.0 & $(13.5)$ & 78.8 & $(15.6)$ & 79.8 & (9.8) & $0.185^{*}$ \\
\hline \multirow{2}{*}{$\begin{array}{ll}\text { Stage } & \text { I } \\
& \text { II }\end{array}$} & 57 & $100.0 \%$ & 16 & $100.0 \%$ & 11 & $100.0 \%$ & 30 & $100.0 \%$ & \\
\hline & 1 & $1.8 \%$ & 0 & $0.0 \%$ & 0 & $0.0 \%$ & 1 & $3.3 \%$ & \\
\hline Tumour size [cm, median (SD)] & 2.6 & (2.5) & 1.8 & (3.8) & 1.8 & (2.0) & 3.5 & $(2.0)$ & $0.2^{*}$ \\
\hline Died of disease & 14 & $24.6 \%$ & 5 & $31.3 \%$ & 3 & $27.3 \%$ & 6 & $20.0 \%$ & $0.309^{\circ}$ \\
\hline Recurrent disease & 28 & $49.1 \%$ & 10 & $62.5 \%$ & 8 & $72.7 \%$ & 9 & $30.0 \%$ & $0.006^{\circ}$ \\
\hline 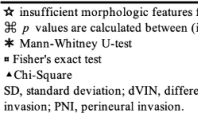 & $\begin{array}{l}\text { N diagn } \\
\text { d (iii) }\end{array}$ & & & & & & & & \\
\hline
\end{tabular}

Results 57 HPV-I p53abn VSCCs, FIGO stage I-II, were evaluated (Table 1). TP53 mutations were identified in 10/11 cases with subtle morphologic abnormalities insufficient for dVIN diagnosis and p53abn IHC extending to margins (synonymous with p53 signature). Positive in situ margins (dVIN and p53 signature) were observed in $27(47.4 \%)$ of cases and significantly associated with disease recurrence $(\mathrm{p}=0.006)$.

Conclusions These findings demonstrate: - p53abn IHC staining at margins, without sufficient morphologic features for dVIN diagnosis, predicts an equivalent or higher recurrence risk compared to morphologic dVIN - p53 IHC is critical for accurate assessment of margin status in HPV-I p53abn VSCC - The need to evaluate biologically-informed treatment approaches for high molecular risk (TP53 mutated) disease in prospective interventional studies

\section{2/\#207 MULTI-OMICS CHARACTERIZATION OF MOLECULAR FEATURES CORRELATED TO PERINEURAL INVASION IN CERVICAL CANCER}

${ }^{1} \mathrm{~T}$ Wan*, ${ }^{2} \mathrm{X}$ Peng, ${ }^{1} \mathrm{H}$ Huang, ${ }^{1} \mathrm{Y}$ Feng, ${ }^{2} \mathrm{H}$ Li, ${ }^{2} \mathrm{X}$ Guo, ${ }^{1} \mathrm{~J}$ Liu. ${ }^{1}$ Sun Yat-sen University Cancer Center, Gynecological Oncology, Guangzhou, China; ${ }^{2}$ Precision Scientific (Beijing) Co., Ltd., Scientific, Beijing, China

\subsection{6/ijgc-2021-IGCS.12}

Objectives Perineural invasion (PNI) is an important pathological feature of cervical cancer, which is often associated with poor prognosis. However, the underlying molecular mechanisms remain unknown.

Methods We performed whole-exome sequencing on 42 pretreatment tumor samples (PNI:20; nonPNI:22) along with the matched blood samples and RNA-seq sequencing on 43 tumor samples (PNI:21, nonPNI:22). We performed an integrated bioinformatic analysis to identify at the gene, pathway, omics and tumor-microenvironment levels to explore the genetic determinants responsible for PNI in cervical cancer.

Results Among 45 known cervical cancer driver genes, we detected 34 in at least one patient in this cohort, including PIK3CA as the most frequently mutated genes (38\%), and followed by KMT2C (19\%). Comparing with nonPNI, PNI tumors harbor significantly more FBXW7 loss-of-function mutations (PNI:6; nonPNI:1, $\mathrm{p}=0.04$ ) and copy-number gain of NKX2-1, PDGFRA (NKX2-1, $\mathrm{p}=0.007$; PDGFRA, $\mathrm{p}=0.04)$. PNI tumors show significantly lower tumor mutation burden than non-PNI ( $p=0.048$ ). We identify 318 genes significantly dysregulated in PNI tumors relative to non-PNI tumors (upregulated: 118; downregulated: 200, $\left|\log _{2} \mathrm{FC}\right|>1$, FDR $<0.25$ ), including downregulation of two tumor-suppressor genes, SOX17 and PTCH1. Interestingly, we find the deactivation of immune-related hallmark pathways in PNI tumors, including interferon_gamma_response, interferon_alpha_response and IL2_STAT5_signaling. Consistently, compared with nonPNI, there are significantly fewer $\mathrm{CD}^{+}$cells in the tumor microenvironment of PNI tumors $(p=0.008)$. Conclusions Loss-of-function mutations in FWXB7 and downregulation of SOX17 and PITH1 are likely responsible for PNI in cervical cancer, and a tumor immunosuppressive environment may also be a contributing factor.

\begin{tabular}{l|l}
\hline 0013/\#573 & TISOTUMAB VEDOTIN (TV) + BEVACIZUMAB OR \\
& PEMBROLIZUMAB OR CARBOPLATIN IN \\
& RECURRENT/METASTATIC CERVICAL CANCER (R/ \\
& MCC): PHASE 1B/2 ENGOT-CX8/G0G-3024/ \\
& INNOVATV 205 STUDY DOSE-ESCALATION \\
& RESULTS
\end{tabular}

${ }^{1} B$ Monk*, ${ }^{*} T$ Van Gorp, ${ }^{3} D$ Lorusso, ${ }^{4} R$ Eilish $O^{\prime}$ Cearbhaill, ${ }^{5} A$ Westermann, ${ }^{6} S$ Banerjee, ${ }^{7} \mathrm{D}$ Collins, ${ }^{8} \mathrm{~J}$ Klat, ${ }^{9} \mathrm{~K}$ Madsen, ${ }^{10} \mathrm{~J}-\mathrm{F}$ Baurain, ${ }^{11} \mathrm{~A}$ Jackson, ${ }^{12}$ I Boere, ${ }^{13} \mathrm{~S}$ Pignata, ${ }^{14} \mathrm{E}$ Gort, ${ }^{15} \mathrm{~J}$ Moroney, ${ }^{16}$ I Soumaoro, ${ }^{17} \mathrm{CM}$ Andreassen, ${ }^{18} \mathrm{~L}$ Nicacio, ${ }^{19} \mathrm{C}$ Gennigens, ${ }^{20}$ I Vergote. ${ }^{1}$ Arizona Oncology (US Oncology Network) University of Arizona, Creighton University, Division of Gynecologic Oncology, Phoenix, USA; ${ }^{2} \mathrm{KU}$ Leuven University Hospitals Leuven, Gynecological Oncology, Leuven, Belgium; ${ }^{3}$ Fondazione Policlinico Universitario Agostino Gemelli IRCCS, Division of Gynecologic Oncology, Rome, Italy; ${ }^{4}$ Memorial Sloan Kettering Cancer Center, Medicine, New York, USA; ${ }^{5}$ Academisch Medisch Centrum, Department of Medical Oncology, Amsterdam, Netherlands; ${ }^{6}$ The Royal Marsden NHS Foundation Trust, Medical Oncology, London, UK; ${ }^{7}$ Cork University Hospital, Oncology Trials Unit, Cork, Ireland; ${ }^{8}$ University Hospital Ostrava, Gynecologic Oncology, Ostrava, Czech Republic; ${ }^{9}$ Righospitalet, University Hospital of Copenhagen, Medical Oncology, Copenhagen, Denmark; ${ }^{10}$ Université Catholique de Louvain (UCLouvain), Medical Oncology, Brussels, Belgium; " University of Cincinnati Cancer Center, Gynecologic Oncology, Cincinnati, USA; ${ }^{12}$ Erasmus MC Cancer Institute, Medical Oncology, Rotterdam, Netherlands; ${ }^{13}$ Instituto Nazionale Tumori IRCCS Fondazione G Pascale, Medical Oncology, Naples, Italy; ${ }^{14}$ University Medical Center Utrecht, Medical Oncology, Utrecht, Netherlands; ${ }^{15}$ University of Chicago, Obstetrics and Gynecology, Chicago, USA; ${ }^{16}$ Genmab US, Inc., Medical Oncology, Princeton, USA; ${ }^{17}$ Genmab, Biostatistics, Copenhagen, Denmark; ${ }^{18}$ Seagen Inc., Medical Oncology, Bothell, USA; ${ }^{19}$ Centre Hospitalier Universitaire de Liege, Medical Oncology, Liege, Belgium; ${ }^{20}$ University of Leuven, Leuven Cancer Institute, Belgium and Luxembourg Gynaecological Oncology Group, Leuven, Belgium

\subsection{6/ijgc-2021-IGCS.13}

Objectives Tisotumab vedotin (TV) monotherapy has shown a manageable and tolerable safety profile with clinically meaningful and durable antitumor activity in previously treated $\mathrm{r} /$ mCC (Lancet Oncol. 2021;22:609-619). The potential of investigational TV combination regimens in $\mathrm{r} / \mathrm{mCC}$ was assessed in a multi-cohort phase $1 \mathrm{~b} / 2$ trial (NCT03786081). Methods Patients with $\mathrm{r} / \mathrm{mCC}$ with progression on/after or ineligible for/intolerant to standard-of-care were treated with escalating doses of TV + bevacizumab (Arm A) or pembrolizumab (Arm B) or carboplatin (Arm C) all given Q3W (figure 1). Primary objectives were to determine recommended phase 2 dosing (RP2D) and evaluate safety/tolerability.

Results A maximum tolerated dose was not reached with any combination. In Arm A, 15 patients received 9 cycles (median) for both TV and bevacizumab. Grade $\geq 3$ adverse events (AEs) occurred in 5 patients (33\%). The RP2D was TV $2.0 \mathrm{mg} / \mathrm{kg}$ + bevacizumab $15 \mathrm{mg} / \mathrm{kg}$ Q3W. Five patients had confirmed objective response (cOR). In Arm B, 13 patients received a median of 5 cycles of TV and 4 cycles of pembrolizumab. Grade $\geq 3$ AEs occurred in 12 patients (92\%). The RP2D was 